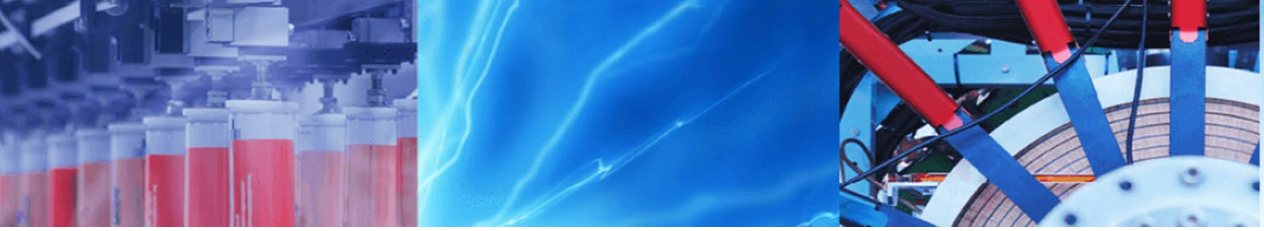

Research Article

\title{
Precoding for multiuser MIMO full-duplex amplify-and-forward relay uplink communication systems
}

\author{
Yunlong Shao ${ }^{1}$ (D) T. A. Gulliver ${ }^{1}$
}

Received: 2 January 2020 / Accepted: 12 March 2020 / Published online: 18 March 2020

(c) Springer Nature Switzerland AG 2020

\begin{abstract}
This paper examines the precoding matrix design for a multiuser multiple-input multiple-output full-duplex (FD) relay uplink communication system with an amplify-and-forward relay. The main problem in FD systems is the loop interference (LI) cancellation, and perfect LI cancellation is not possible so residual LI exists. Linear precoders are used at the sources and relay, and a minimum mean-squared-error receiver is employed at the destination to reduce the influence of the residual LI. An iterative method is developed to solve the highly nonconvex joint source, relay, and receiver optimization problem. Simulation results are presented which show that the proposed iterative optimization algorithm provides better performance than existing half duplex systems in terms of the achievable rate even with residual LI.
\end{abstract}

Keywords Amplify-and-forward $\cdot$ Achievable rate $\cdot$ Full-duplex relaying $\cdot$ MIMO relay precoding

\section{Introduction}

Multiple transmit and receive antenna wireless communication systems, known as MIMO (multiple-input multipleoutput) systems, were first devised in the 1970s [1]. Subsequently, MIMO technology has been shown to significantly improve the spectral and energy efficiency of wireless systems. Complex wireless propagation environments include multipath fading, shadowing and interference which cause errors at the destination receiver. To reduce these errors and improve performance, relay assisted cooperative communication systems have been developed [2]. There has been significant research on one way relay communication systems. In [3], an iterative algorithm was developed to design the source and relay precoders and destination combiner for a single-user MIMO relay system. The joint design of source and relay precoding in a two-way relay system where both the source and relay nodes are equipped with multiple antennas was presented in [4]. In [5], joint transceiver optimization for a multiuser MIMO relay uplink communication system was studied.

In [3-5], the signal is transmitted via the source to relay and then the relay to destination links. Since source to destination transmission takes two time slots, the achievable rate is reduced by half. Full duplex (FD) MIMO relaying has been proposed to increase the achievable rate compared to conventional half-duplex (HD) systems [6]. The loop interference (LI) is a critical issue in FD systems because the relay transmits and receives simultaneously. In general, the $\mathrm{LI}$ is much larger than the channel noise and so can degrade the system performance significantly. Therefore, LI cancellation techniques have been developed [7]. Temporal cancellation methods such as antenna isolation and analog/digital precancellation have been shown to be effective $[9,10]$. However, it is impossible to cancel the $\mathrm{LI}$ completely, and the residual $\mathrm{LI}$ can still be larger than the noise level.

In [11], it was shown that performance improvements over HD systems can only be achieved using linear

$\triangle$ Yunlong Shao, yshao@uvic.ca| 1 Department of Electrical and Computer Engineering, University of Victoria, Victoria, BC V8W 2Y2, Canada. 
precoders and receiver combiners, and $\mathrm{LI}$ reduction at the relay is preferable to pre-cancellation at the relay transmitter. A closed-form solution for the covariance matrix of the transmitted signal at the relay was derived which enables the performance evaluation of a wide range of transceivers. Linear source and relay precoders and destination combiner for MIMO FD relay communication systems was investigated in [12]. The effect of residual LI was explored and two iterative algorithms were proposed to minimize the MSE. In contrast to previous work which focused on MSE minimization, in [13] spatial mitigation of the LI at the relay node was considered, an a joint precoding/decoding design that maximizes the end-to-end (e2e) performance was proposed. Moreover, an optimal antena selection (AS) scheme that maximizes the e2e SNR at the destination was given along with sub-optimal AS schemes. While results exists on precoding design for one-way MIMO FD relay system, to the best of our knowledge precoding design for multiuser MIMO FD AF relay communication systems has not been considered.

This paper examines a MIMO full duplex relaying uplink communication system with multiple users. Precoder design for the users and relay, and the linear combiner at the destination are investigated considering the residual LI. Further, multiple antennas are assumed at all nodes. The design is based on minimizing the MSE of the received signal at the destination. An iterative method is developed to update the source and relay precoding matrices and the linear combining matrix at the destination.

The remainder of this paper is organized as follows. Section 1 describes the system model and the solution of the optimization problem is given in Sect. 3. Simulation results are presented in Sect. 4 to demonstrate the effectiveness of this solution, and finally some concluding remarks are given in Sect. 5.

Notation The following notation is used. Bold uppercase, bold lowercase and normal letters denote matrices, vectors and scalars, respectively. vec(.) denotes the vectorization operation of the matrix and $\otimes$ denotes the matrix Kronecker product. $\operatorname{tr}\{\cdot\}$ is the trace of a matrix, and $\mathbf{I}_{N}$ is the $N \times N$ identity matrix.

\section{System model}

We consider a MIMO full-duplex (FD) relay system where direct link communications between the source and destination is negligible due to large-scale fading and the long distance between the two nodes.

As shown in Fig. 1, $K$ users transmit to the destination with the help of a FD relay. The ith user and the destination are equipped with $N_{i}$ and $N_{d}$ antennas, respectively. The FD relay node is equipped with $N_{r}$ and $N_{t}$ antennas

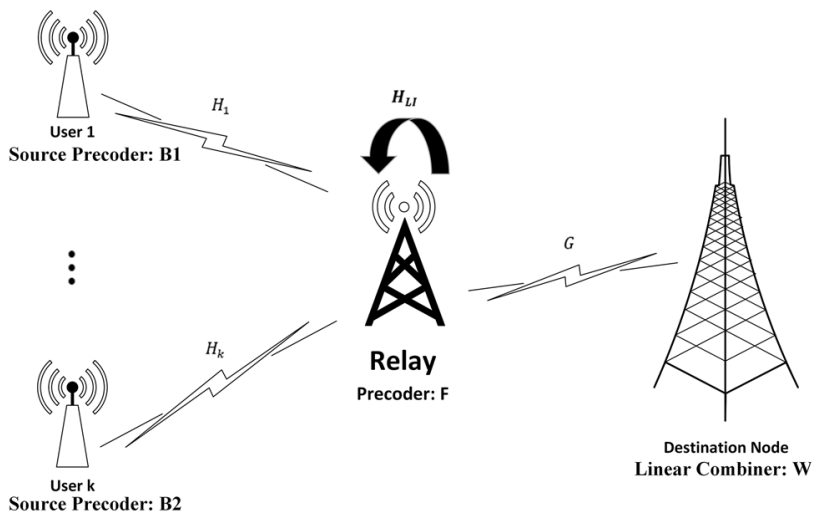

Fig. 1 The multiuser MIMO full-duplex (FD) relay system model

for receiving and transmitting simultaneously. Let $\mathbf{s}_{i}[n]$ denote the $N_{i} \times 1$ signal vector at time $n$ for the $i$ th source. The number of independent data streams from all users is $N_{b}=\sum_{i=1}^{K} N_{i}$. Without loss of generality, we assume $N_{b} \leq \min \left(N_{r}, N_{t}, N_{d}\right)$. A linear precoding matrix $\mathbf{B}_{i}$ is applied to the signal vector $\mathrm{s}_{i}$ before transmission. The received signal at the relay can be expressed as

$\mathrm{y}_{r}[n]=\sum_{i=1}^{K} \mathbf{H}_{i}[n] \mathbf{B}_{i}[n] \mathrm{s}_{i}[n]+\mathbf{H}_{L l}[n] \mathrm{t}[n]+\mathbf{n}_{r}[n]$,

where $\mathbf{H}_{i}[n] \in \mathbb{C}^{N_{r} \times N_{i}}$ is the channel matrix between the $i$ th user and relay, $\mathbf{n}_{r} \in \mathbb{C}^{N_{r} \times 1}$ is the noise vector at the relay and $\mathbf{H}_{L l} \in \mathbb{C}^{N_{r} \times N_{t}}$ is the loop interference (LI) channel matrix.

Equation (1) shows that the signal received at the relay is corrupted by LI, but loop interference cancellation techniques can be employed. Then from [7], (1) can be rewritten as

$\mathrm{y}_{r}[n]=\sum_{i=1}^{K} \mathbf{H}_{i}[n] \mathbf{B}_{i}[n] \mathrm{s}_{i}[n]+\mathbf{H}_{L /}[n] \mathrm{t}[n]+\mathbf{T}[n]+\mathbf{n}_{r}[n]$,

where $\mathbf{T}[n]=-\mathbf{H}_{L /}[n] \mathrm{t}[n]$ if the $\mathrm{LI}$ cancellation is perfect. However, in practical communication systems $\mathbf{T}[n]=-\mathbf{H}_{L /}[n] \tilde{\mathrm{t}}[n]$, where $\tilde{\mathrm{t}}[n]$ is a noisy version of $\mathrm{t}[n]$, so that

$\mathbf{y}_{r}[n]=\sum_{i=1}^{K} \mathbf{H}_{i}[n] \mathbf{B}_{i}[n] \mathrm{s}_{i}[n]+\mathbf{H}_{L /}[n] \Delta \mathrm{t}[n]+\mathbf{n}_{r}[n]$,

where $\Delta \mathrm{t}[n]=\mathrm{t}[n]-\tilde{\mathrm{t}}[n]$ and $\mathbf{H}_{L I}[n] \Delta \mathrm{t}[n]$ is the residual $\mathrm{LI}$ after imperfect loop interference cancellation.

At time $n+1$, the full duplex relay multiplies the received signal with a relay precoder $\mathbf{F}[n+1] \in \mathbb{C}^{N_{r} \times N_{t}}$ and immediately transmits this signal to the destination. The signal at the destination can be expressed as 


$$
\begin{aligned}
\mathbf{y}_{d}[n]= & \mathbf{G}[n+1] \mathbf{F}[n+1] \overline{\mathbf{H}}[n] \mathbf{s}[n] \\
& \left.+\mathbf{G}[n+1] \mathbf{F}_{[} n+1\right] \mathbf{H}_{L /}[n] \Delta \mathrm{t}[n] \\
& +\mathbf{G}[n+1] \mathbf{F}[n+1] \mathbf{n}_{r}[n]+\mathbf{n}_{d}[n+1] .
\end{aligned}
$$

where $\overline{\mathbf{H}}[n]=\left[\mathbf{H}_{1}[n] \mathbf{B}_{1}[n], \ldots, \mathbf{H}_{K}[n] \mathbf{B}_{K}[n]\right]$ is the equivalent multiple access MIMO channel matrix of the source to relay link, and $\mathbf{s}[n]=\left[\mathrm{s}_{1}[n]^{T}, \ldots, \mathrm{s}_{k}[n]^{T}\right]^{T}$ is the equivalent transmitted signal vector where (.) ${ }^{T}$ denotes vector transpose. Let $\mathbb{E}\left[\mathbf{s s}^{H}\right]=\mathbf{I}_{N_{b}}$ where $(.)^{H}$ denotes matrix Hermitian transpose and $\mathbb{E}[$.] denotes expectation. We assume that the channel variation during the precoder update interval is relatively small and so can be be ignored. Thus, the time index has no influence on the precoder design. For simplicity, this index is omitted to obtain a more concise expression for the received signal in (4) which is

$$
\begin{aligned}
\mathbf{y}_{d} & =\mathbf{G F} \overline{\mathbf{H}} \mathbf{s}+\mathbf{n} \\
& =\mathbf{H s}+\mathbf{n},
\end{aligned}
$$

w h e r e

$$
\mathbf{H}=\left[\mathrm{GFH}_{1} \mathbf{B}_{1}, \ldots, \mathrm{GFH}_{k} \mathbf{B}_{K}\right],
$$

$\mathbf{n}=\mathbf{G F H}_{L I} \Delta \mathrm{t}+\mathbf{G F n}_{r}+\mathbf{n}_{d}$ is the equivalent noise vector, $\mathbf{G}$ is the $N_{d} \times N_{t}$ MIMO channel matrix between the relay and destination, and $\mathbf{n}_{d} \in \mathbb{C}^{N_{d} \times N_{b}}$ is the noise vector at the destination. $\Delta \mathrm{t}$ can be modeled as white Gaussian noise [8] which is independent of $\mathbf{n}_{r}$ and $\mathbf{n}_{d}$. The corresponding covariance matrix of $\mathbf{n}$ can be expressed as

$\mathbf{C}_{n}=\mathbb{E}\left[n n^{H}\right]=\sigma_{t}^{2} \mathbf{G F H} \mathbf{H}_{L I} \mathbf{H}_{L I}^{H} \mathbf{F}^{H} \mathbf{G}^{H}+\mathbf{G F F} \mathbf{F}^{H} \mathbf{G}^{H}+\mathbf{I}_{N_{D^{\prime}}}$

where $\sigma_{t}^{2}$ is the variance of $\Delta \mathrm{t}$.

A linear combiner matrix $\mathbf{W} \in \mathbb{C}^{N_{d} \times N_{b}}$ is applied on the received signal at the destination and the resulting estimated signal is

$\hat{s}=\mathbf{W}^{H} \mathbf{y}_{d}$.

The mean squared error can be expressed as

$\operatorname{MSE}(\mathbf{B}, \mathbf{F}, \mathbf{W})=\mathbb{E}\left[(\hat{\mathbf{s}}-\mathbf{s})(\hat{\mathbf{s}}-\mathbf{s})^{H}\right]$

The optimization problem is to minimize the mean square error of the received signal at the destination under the constraints of individual user power and relay power budget, which can be expressed as

$$
\min _{\mathbf{B}, \mathbf{W}, \mathbf{W}} \operatorname{tr}(\mathbf{M S E})
$$

$$
\text { s.t.tr }\left(\mathbf{F}\left(\sum_{i=1}^{K} \mathbf{H}_{i} \mathbf{B}_{i} \mathbf{B}_{i}^{H} \mathbf{H}_{i}^{H}+\sigma_{t}^{2} \mathbf{H}_{L I} \mathbf{H}_{L I}^{H}+\mathbf{I}_{N_{r}}\right) \mathbf{F}^{H}\right) \leq P_{r}
$$

$\operatorname{tr}\left(\mathbf{B}_{i} \mathbf{B}_{i}^{H}\right) \leq P_{s_{i}} i=1, \ldots, K$

\section{Solution of the optimization problem}

The problem in (9) is highly non-convex, which makes determining an optimal solution difficult. Thus in this section, an iterative algorithm is developed to solve this optimization problem. The source precoding matrix $\mathbf{B}_{i}$, relay precoding matrix $\mathbf{F}$ and destination combiner matrix $\mathbf{W}$ are determined to minimize the mean-squared-error (MSE) of the signal estimate

$$
\begin{aligned}
\mathbf{M S E} & =\operatorname{tr}\left(\mathbb{E}\left[(\hat{\mathbf{s}}-\mathbf{s})(\hat{\mathbf{s}}-\mathbf{s})^{H}\right]\right) \\
& =\operatorname{tr}\left(\left(\mathbf{W}^{H} \mathbf{H}-\mathbf{I}_{N_{b}}\right)\left(\mathbf{W}^{H} \mathbf{H}-\mathbf{I}_{N_{b}}\right)^{H}+\mathbf{W}^{H} \mathbf{C}_{n} \mathbf{W}\right),
\end{aligned}
$$

A closed form solution for the optimization problem in (10) is intractable. Therefore, an iterative algorithm is used to solve this problem by introducing three convex subproblems.

Given the relay precoder $\mathbf{F}$ and ith source precoder $\mathbf{B}_{i}$, the original optimization problem becomes an unconstrained problem. The destination combiner can then be obtained by taking the derivative of the MSE with respect to $\mathbf{W}$, and the solution can be expressed as

$\mathbf{W}=\left(\mathbf{H H}^{H}+\mathbf{C}_{n}\right)^{-1} \mathbf{H}$.

Next, given the linear combiner $\mathbf{W}$ and $\mathbf{B}_{i}, \mathbf{F}$ can be obtained by solving the following problem

$\min _{\overline{\mathbf{F}}} \operatorname{tr}\left(\left(\overline{\mathbf{G}} \mathbf{F} \overline{\mathbf{H}}-\mathbf{I}_{N_{b}}\right)\left(\overline{\mathbf{G}} \mathbf{F} \overline{\mathbf{H}}-\mathbf{I}_{N_{b}}\right)^{H}+\overline{\mathbf{G}} \mathbf{F} \mathbf{F}^{H} \overline{\mathbf{G}}^{H}\right)$

s.t. $\operatorname{tr}\left(\mathbf{F}\left(\overline{\mathbf{H}} \overline{\mathbf{H}}^{H}+\sigma_{t}^{2} \mathbf{H}_{L I} \mathbf{H}_{L I}^{H}+\mathbf{I}_{N_{r}}\right) \mathbf{F}^{H}\right) \leq P_{r}$

where $\overline{\mathbf{G}}=\mathbf{W}^{H} \mathbf{G}$ and $\overline{\mathbf{H}}=\left[\mathbf{H}_{1} \mathbf{B}_{1}, \ldots, \mathbf{H}_{k} \mathbf{B}_{k}\right]$. The convexity of the problem in (12) was proven in [5].

Similar to the result in [5], the KKT conditions can be applied to the convex subproblem in (12). Then the optimal relay precoder can be expressed as

$\mathbf{F}=\overline{\mathbf{G}}^{H}\left(\overline{\mathbf{G}} \overline{\mathbf{G}}^{H}+\mu \mathbf{I}_{N_{b}}\right)^{-1} \overline{\mathbf{H}}^{H}\left(\overline{\mathbf{H}} \overline{\mathbf{H}}^{H}+\sigma_{t}^{2} \mathbf{H}_{L l} \mathbf{H}_{L l}^{H}+\mathbf{I}_{N_{r}}\right)^{-1}$,

where $\mu \geq 0$ is the Lagrange multiplier which can be found from the complementary slackness condition given by

$\mu\left(\operatorname{tr}\left(\mathbf{F}\left(\overline{\mathbf{H}} \overline{\mathbf{H}}^{H}+\sigma_{t}^{2} \mathbf{H}_{L I} \mathbf{H}_{L I}^{H}+\mathbf{I}_{N_{r}}\right) \mathbf{F}^{H}\right)-P_{r}\right)=0$.

If $\mu=0$, we have from (15) that

$\mathbf{F}=\overline{\mathbf{G}}^{H}\left(\overline{\mathbf{G}} \overline{\mathbf{G}}^{H}\right)^{-1} \overline{\mathbf{H}}^{H}\left(\overline{\mathbf{H}} \overline{\mathbf{H}}^{H}+\sigma_{t}^{2} \mathbf{H}_{L I} \mathbf{H}_{L I}^{H}+\mathbf{I}_{N_{r}}\right)^{-1}$,

Since in this case $\mu=0$ already satisfies $\mu \geq 0$, if $\mathbf{F}$ in (15) satisfies the constraint in (12b), then (15) is a solution to the problem in (12). Otherwise, if $\mu>0$, then 
$\operatorname{tr}\left(\mathbf{F}\left(\overline{\mathbf{H}} \overline{\mathbf{H}}^{H}+\sigma_{t}^{2} \mathbf{H}_{L I} \mathbf{H}_{L l}^{H}+\mathbf{I}_{N_{r}}\right) \mathbf{F}^{H}\right) \leq P_{r}$.

To find $\mu$, substitute (15) into (16) and solve the following nonlinear equation

$$
\begin{aligned}
\operatorname{tr}\left(\overline{\mathbf{G}}^{H}\left(\overline{\mathbf{G}} \overline{\mathbf{G}}^{H}+\mu \mathbf{I}_{N_{b}}\right)^{-1} \overline{\mathbf{H}}^{H}\left(\overline{\mathbf{H}} \overline{\mathbf{H}}^{H}+\sigma_{t}^{2} \mathbf{H}_{L l} \mathbf{H}_{L I}^{H}+\mathbf{I}_{N_{r}}\right)^{-1}\right. \\
\left.\times \overline{\mathbf{H}}\left(\overline{\mathbf{G}} \overline{\mathbf{G}}^{H}+\mu \mathbf{I}_{N_{b}}\right)^{-1} \overline{\mathbf{G}}\right)=P_{r} .
\end{aligned}
$$

Using the singular value decomposition (SVD) of $\overline{\mathbf{G}}$ given by $\mathbf{U} \Sigma \mathbf{V}^{H}$ where $\mathbf{U} \in \mathbb{C}^{N_{b} \times N_{b}}$ and $\mathbf{V} \in \mathbb{C}^{N_{t} \times N_{t}}$ are unitary matrices, $\Sigma=\left[\begin{array}{ll}\mathbf{O} & 0 \\ 0 & 0\end{array}\right]_{N_{b} \times N_{t}}$, and $\mathbf{O}=\operatorname{diag}\left\{\sigma_{1}, \sigma_{2}, \ldots, \sigma_{L}\right\}$, (17) can be expressed as

$$
\begin{aligned}
& \operatorname{tr}\left(\Sigma\left(\Sigma^{2}+\mu \mathbf{I}_{N_{b}}\right)^{-1} \mathbf{U}^{H} \overline{\mathbf{H}}^{H}\left(\overline{\mathbf{H}} \overline{\mathbf{H}}^{H}+\sigma_{t}^{2} \mathbf{H}_{L l} \mathbf{H}_{L I}^{H}+\mathbf{I}_{N_{r}}\right)^{-1}\right. \\
& \left.\quad \times \overline{\mathbf{H}} \mathbf{U}\left(\Sigma^{2}+\mu \mathbf{I}_{N_{b}}\right)^{-1} \Sigma\right)=P_{r} .
\end{aligned}
$$

This can be shown to be equivalent to [5]

$$
\sum_{i=1}^{N_{b}} \frac{\sigma_{i}^{2} \gamma_{i}}{\left(\sigma_{i}^{2}+\mu\right)^{2}}=P_{r}
$$

where $\sigma_{i}$ and $\gamma_{i}$ are the main diagonal elements of $\Sigma$ and $\Gamma$, respectively. We have that $\Gamma=\mathbf{U}^{H} \overline{\mathbf{H}}^{H}\left(\overline{\mathbf{H}} \overline{\mathbf{H}}^{H}+\sigma_{t}^{2} \mathbf{H}_{L I} \mathbf{H}_{L I}^{H}+\mathbf{I}_{N_{r}}\right)^{-1} \overline{\mathbf{H}} \mathbf{U}$. A technique such as the bisection method can be used to find $\mu$ since the left hand side of (19) is monotonically decreasing with respect to $\mu$ [15].

In the third subproblem, the source precoder $\mathbf{B}_{i}$ can be determined using $\mathbf{W}$ and $\mathbf{F}$ obtained above. The optimization problem can be reformulated as a convex quadratically constrained quadratic program (QCQP) problem using the following steps. Using the matrix identities

$\left(\mathbf{C}^{T} \mathbf{D}\right)=(\operatorname{vec}(\mathbf{C}))^{T} \operatorname{vec}(\mathbf{D})$,

$\operatorname{tr}(\mathbf{A B})=\operatorname{tr}(\mathbf{B A})$

$\operatorname{vec}(\mathbf{C D})=(\mathbf{I} \otimes \mathbf{C}) \operatorname{vec}(\mathbf{D})$,

in (12) and $\mathbf{A}_{i}=\mathbf{W}^{H} \mathbf{G F H}_{i}$, we have

$$
\begin{aligned}
\operatorname{tr}\left(\mathbf{A}_{i} \mathbf{B}_{i} \mathbf{B}_{i}^{H} \mathbf{A}_{i}^{H}\right) & =\left(\operatorname{vec}\left(\mathbf{B}_{i}\right)\right)^{H} \operatorname{vec}\left(\mathbf{A}_{i}^{H} \mathbf{A}_{i} \mathbf{B}_{i}\right) \\
& =\left(\operatorname{vec}\left(\mathbf{B}_{i}\right)\right)^{H}\left(\mathbf{I}_{N_{b}} \otimes\left(\mathbf{A}_{i}^{H} \mathbf{A}_{i}\right)\right)\left(\operatorname{vec}\left(\mathbf{B}_{i}\right)\right) \\
& =\mathbf{b}_{i}^{H}\left(\mathbf{I}_{N_{b}} \otimes\left(\mathbf{A}_{i}^{H} \mathbf{A}_{i}\right)\right) \mathbf{b}_{i},
\end{aligned}
$$

and

$$
\begin{aligned}
\operatorname{tr} & \left(\mathbf{W}^{H} \mathbf{G F}\left[\mathbf{H}_{1} \mathbf{B}_{1}, \ldots, \mathbf{H}_{K} \mathbf{B}_{K}\right]\right) \\
& =\operatorname{tr}\left(\left[\mathbf{A}_{1} \mathbf{B}_{1}, \ldots, \mathbf{A}_{K} \mathbf{B}_{K}\right]\right) \\
& =\sum_{i=1}^{K} \operatorname{tr}\left(\mathbf{A}_{i i} \mathbf{B}_{i}\right)=\sum_{i=1}^{K}\left(\operatorname{vec}\left(\mathbf{A}_{i i}^{T}\right)\right)^{T} \mathbf{b}_{i}
\end{aligned}
$$

where $\mathbf{b}_{i}=\operatorname{vec}\left(\mathbf{B}_{i}\right)$ and $\mathbf{A}_{i i}$ is a matrix containing rows $\left(\sum_{j=1}^{i-1} N_{j}+1\right)$ to $\left(\sum_{j=1}^{i-1} N_{j}\right)$ of $\mathbf{A}_{i}$. The MSE can be written as

$$
\begin{aligned}
\mathbf{M S E}= & \operatorname{tr}\left(\left(\mathbf{W}^{H} \mathbf{G F}\left(\sum_{i=1}^{K} \mathbf{H}_{i} \mathbf{B}_{i} \mathbf{B}_{i}^{H} \mathbf{H}_{i}^{H}\right) \mathbf{F}^{H} \mathbf{G}^{H} \mathbf{W}\right.\right. \\
& -\mathbf{W}^{H} \mathbf{G F}\left[\mathbf{H}_{1} \mathbf{B}_{1}, \ldots, \mathbf{H}_{K} \mathbf{B}_{K}\right] \\
& -\left(\mathbf{W}^{H} \mathbf{G F}\left[\mathbf{H}_{1} \mathbf{B}_{1}, \ldots, \mathbf{H}_{K} \mathbf{B}_{K}\right]\right)^{H} \\
& +\mathbf{I}_{N_{b}}+\mathbf{W}^{H}\left(\sigma_{t}^{2} \mathbf{G F} \mathbf{H}_{L /} \mathbf{H}_{L I}^{H} \mathbf{F}^{H} \mathbf{G}^{H}\right. \\
& \left.+\mathbf{G F F}^{H} \mathbf{G}^{H}+\mathbf{I}_{N_{b}}\right) \mathbf{W} \\
= & \sum_{i=1}^{K} \mathbf{b}_{i}^{H}\left(\mathbf{I}_{N_{i}} \otimes\left(\mathbf{A}_{i}^{H} \mathbf{A}_{i}\right)\right) \mathbf{b}_{i}-\sum_{i=1}^{K}\left(\operatorname{vec}\left(\mathbf{A}_{i i}^{T}\right)\right)^{T} \mathbf{b}_{i} \\
& -\sum_{i=1}^{K} \mathbf{b}_{i}^{H} \operatorname{vec}\left(\mathbf{A}_{i i}^{H}\right)+t \\
= & \mathbf{b}^{H} \mathbf{A} \mathbf{b}-\mathbf{c}^{H} \mathbf{b}-\mathbf{b}^{H} \mathbf{c}+t,
\end{aligned}
$$

where

$\mathbf{t}=\operatorname{tr}\left(\mathbf{I}_{N_{b}}+\mathbf{W}^{H}\left(\sigma_{t}^{2} \mathbf{G F} \mathbf{H}_{L I} \mathbf{H}_{L I}^{H} \mathbf{F}^{H} \mathbf{G}^{H}\right.\right.$

$\left.\left.+\mathbf{G F F}^{H} \mathbf{G}^{H}+\mathbf{I}_{N_{d}}\right) \mathbf{W}\right)$,

$\mathbf{A}=b d\left(\mathbf{I}_{N_{1}} \otimes\left(\mathbf{A}_{1}^{H} \mathbf{A}_{1}\right), \ldots, \mathbf{I}_{N_{k}} \otimes\left(\mathbf{A}_{K}^{H} \mathbf{A}_{K}\right)\right)$,

$\mathbf{b}=\left[b_{1}^{T}, \ldots, b_{K}^{T}\right]^{T}$,

$\mathbf{c}=\left[\left(\operatorname{vec}\left(\mathbf{A}_{11}^{H}\right)\right)^{T}, \ldots,\left(\operatorname{vec}\left(\mathbf{A}_{K K}^{H}\right)\right)^{T}\right]^{T}$.

The MSE can be equivalently expressed as

$$
\begin{aligned}
\mathbf{M S E}= & \mathbf{b}^{H} \mathbf{A}^{\frac{1}{2}} \mathbf{A}^{\frac{1}{2}} \mathbf{b}-\mathbf{c}^{H} \mathbf{A}^{-\frac{1}{2}} \mathbf{A}^{\frac{1}{2}} \mathbf{b}-\mathbf{b}^{H} \mathbf{A}^{\frac{1}{2}} \mathbf{A}^{-\frac{1}{2}} \mathbf{c} \\
& +\mathbf{c}^{H} \mathbf{A}^{-\frac{1}{2}} \mathbf{A}^{-\frac{1}{2}} \mathbf{c}-\mathbf{c}^{H} \mathbf{A}^{-1} \mathbf{c}+t \\
= & \left(\mathbf{b}^{H} \mathbf{A}^{\frac{1}{2}}-\mathbf{c}^{H} \mathbf{A}^{-\frac{1}{2}}\right)\left(\mathbf{A}^{\frac{1}{2}} \mathbf{b}-\mathbf{A}^{-\frac{1}{2}} \mathbf{c}\right)-\mathbf{c}^{H} \mathbf{A}^{-1} \mathbf{c}+t,
\end{aligned}
$$

where $\mathbf{A}^{\frac{1}{2}} \mathbf{A}^{\frac{1}{2}}=\mathbf{A} \mathbf{A}^{\frac{1}{2}}=\mathbf{A}^{\frac{H}{2}}$. Note that the term $\mathbf{c}^{H} \mathbf{A}^{-1} \mathbf{c}+t$ in (23) can be eliminated since it is not related to $\mathbf{b}$.

The original problem in (10) is equivalent to the following QCQP problem

$$
\min _{b}\left(\mathbf{A}^{\frac{1}{2}} \mathbf{b}-\mathbf{A}^{-\frac{1}{2}} \mathbf{c}\right)^{H}\left(\mathbf{A}^{\frac{1}{2}} \mathbf{b}-\mathbf{A}^{-\frac{1}{2}} \mathbf{c}\right)
$$

s.t. $\mathbf{b}^{H} \mathbf{C b} \leq \overline{P_{r}}$

$\mathbf{b}^{H} \mathbf{D b} \leq P_{s_{i}}, i=1, \ldots, K$

where $\bar{P}_{r}=P_{r}-\operatorname{tr}\left(\mathbf{F}\left(\sigma_{t}^{2} \mathbf{H}_{L l} \mathbf{H}_{L I}^{H}+\mathbf{I}_{N_{r}}\right) \mathbf{F}^{H}\right), \quad \mathbf{C}_{i}=\mathbf{F H}_{i}$, $\mathbf{C}=b d\left(\mathbf{I}_{N_{1}} \otimes\left(\mathbf{C}_{1}^{H} \mathbf{C}_{1}\right), \ldots, \mathbf{I}_{N_{1}} \otimes\left(\mathbf{C}_{K}^{H} \mathbf{C}_{K}\right)\right) \quad$ a $\mathrm{n} \mathrm{d}$ $\mathbf{D}_{i}=b d\left(\mathbf{D}_{i 1}, \mathbf{D}_{i 2}, \ldots, \mathbf{D}_{i K}\right) \quad$ with $\quad \mathbf{D}_{i i}=\mathbf{I}_{N_{i}}$ a nd $\mathbf{D}_{i j}=0, j=1, \ldots, K, j \neq i$. A QCQP problem can be solved efficiently using the disciplined convex programming toolbox CVX [16]. A proof of the convexity of a problem similar to (24a) was given in [5]. 


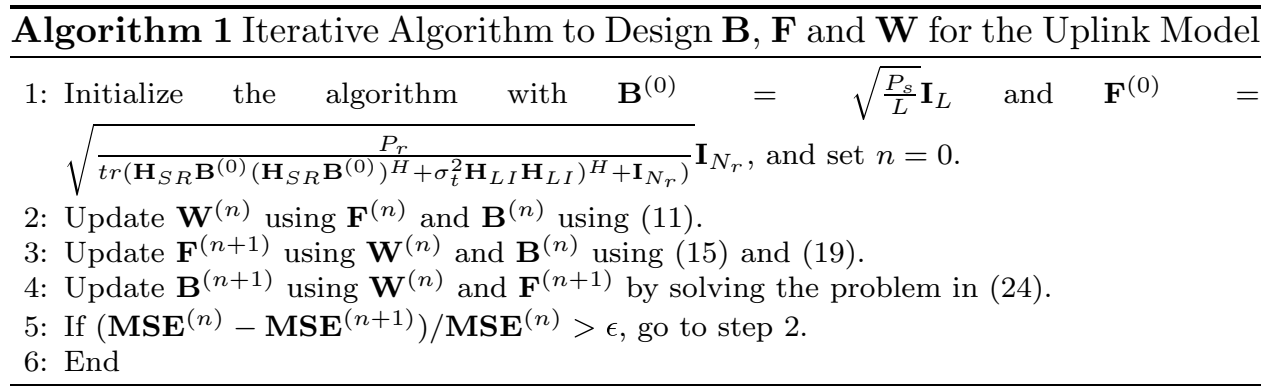

\section{Numerical results}

In this section, the performance of the proposed multiuser MIMO FD relay algorithm is evaluated and compared with that of the corresponding half-duplex (HD) relay system using numerical simulation. The HD algorithm is the same as the FD algorithm but with no LI. The achievable rate of the HD system is half that of the FD system without residual $\mathrm{LI}$ since it requires two time slots for source nodes to destination data transmission. For simplicity, we consider a system with two source users. The extension to more than two users is straightforward. We assume the source users are equipped with two antennas and the destination node has four antennas. The relay node is equipped with four receive antennas and four transmit antennas. As in the related literature, flat-fading MIMO channels are considered. It is assumed that the entries of $\mathbf{H}_{i}, \mathbf{G}$ and $\mathbf{H}_{L /}$ are independent and identically distributed (i.i.d.) complex Gaussian random variables with zero mean and unit variance. Further, all

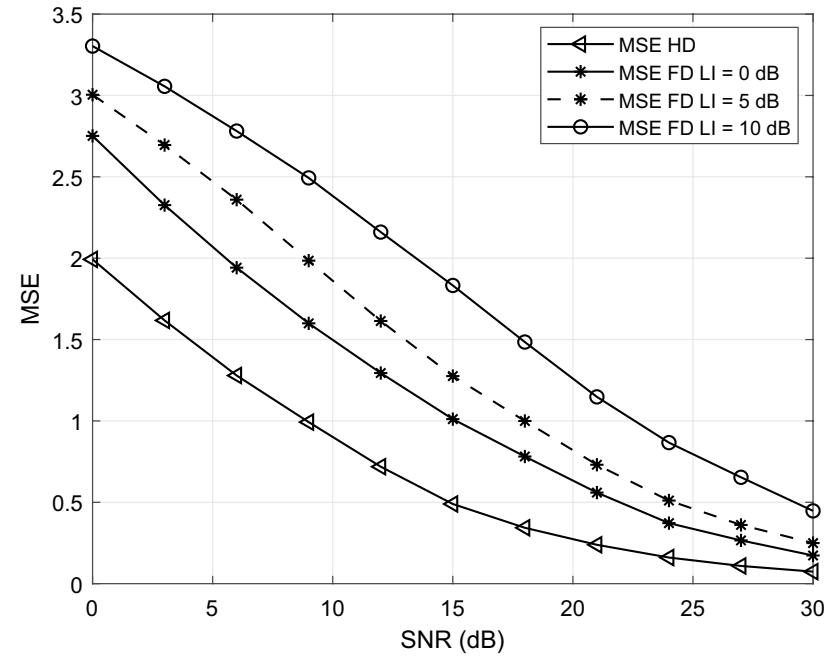

Fig. 2 MSE for the HD system and FD system with different residual LI levels noise terms are i.i.d. complex circularly symmetric Gaussian random variables with zero mean and unit variance. The signal to noise ratio (SNR) of the relay to destination link is fixed at $30 \mathrm{~dB}$ while the SNR of the source to relay link varies from $0 \mathrm{~dB}$ to $30 \mathrm{~dB}$. As discussed in [9], the residual $\mathrm{LI}$ magnitude is from $0 \mathrm{~dB}$ to $30 \mathrm{~dB}$. Therefore, the residual $\mathrm{LI}$ is set to $0 \mathrm{~dB}, 5 \mathrm{~dB}$ and $10 \mathrm{~dB}$ here. In all cases, the average results for 1000 independent channel realizations are given.

Figure 2 presents the MSE for a conventional half duplex system and a full duplex system with different levels of residual LI. The convergence tolerance is set to $\epsilon=0.00001$ and the maximum number of iterations is 30 . It is clear that the HD system provides the best MSE performance. The FD system has a higher MSE than the HD system due to the existence of residual LI. Further, the FD MSE performance is degraded as the residual LI level increases.

The mutual information for the system in (5) can be obtained using an approach similar to that in [14], which gives

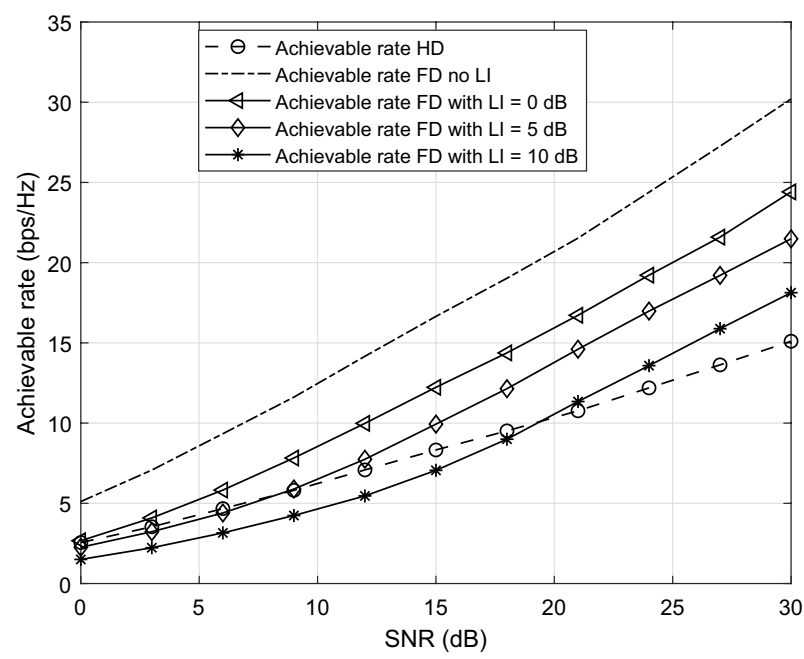

Fig. 3 Achievable rate of the HD system and FD system with different levels of residual LI 


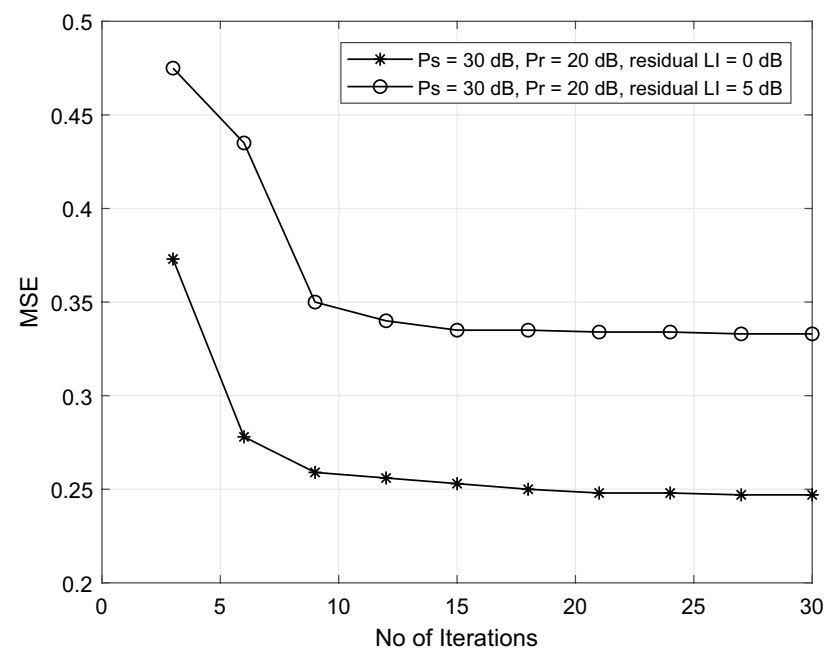

Fig. 4 Converge rate of the proposed FD system with different residual $\mathrm{LI}$ levels

$$
\begin{aligned}
I(y ; x)= & \log _{2} \operatorname{det}\left[\mathbf{I}_{N_{d}}+(\mathbf{G F} \overline{\mathbf{H}})(\mathbf{G F} \overline{\mathbf{H}})^{H}\right. \\
& \left.\times\left(\sigma_{t}^{2} \mathbf{G F H}_{L I} \mathbf{H}_{L I}^{H} \mathbf{F}^{H} \mathbf{G}^{H}+\mathbf{G F F}^{H} \mathbf{G}^{H}+\mathbf{I}_{N_{d}}\right)^{-1}\right] .
\end{aligned}
$$

Figure 3 shows the achievable rate of the HD and FD systems. This shows that the FD system achievable rate is twice that of the HD system if the $\mathrm{LI}$ is canceled completely. Further, the FD system achievable rate is higher than that of the HD system when the residual LI magnitude is $0 \mathrm{~dB}$ and $5 \mathrm{~dB}$. However, when the residual LI magnitude is $10 \mathrm{~dB}$, the achievable rate of the FD system is better only when the source user SNR is greater than $20 \mathrm{~dB}$.

Figure 4 presents the number of iterations required for convergence with the proposed FD system. This shows that convergence to an optimal solution occurs after 12 iterations. Further, the MSE performance is significantly improved after 6 iterations. The small number of iterations required indicates that the complexity of the proposed design algorithm is reasonable.

\section{Conclusion}

In this paper, the precoder design for the uplink of a non-regenerative MIMO full duplex (FD) relay multiuser system with residual loop interference (LI) was investigated. The linear source and relay precoding matrices and the destination combining matrix were optimized to minimize the mean squared error (MSE). The original non-convex problem was converted into three convex subproblems and an iterative algorithm was developed to optimize the source, relay and destination precoder matrices. Simulation results were presented which confirm that the proposed iterative method outperforms the corresponding half duplex (HD) relaying scheme in terms of achievable rate when there is no residual LI. It was shown that the achievable rate is degraded as the residual $\mathrm{LI}$ increases.

\section{Compliance with ethical standards}

Conflict of interest On behalf of all authors, the corresponding author states that there is no conflict of interest.

\section{References}

1. Kaye A, George D (1970) Transmission of multiplexed PAM signals over multiple channel and diversity systems. IEEE Trans Commun Technol 18(5):520-526

2. Wang R, Tao M, Huang $Y$ (2012) Linear precodig designs for amplify-and-forward multiuser two-way relay systems. IEEE Trans Wireless Commun 11(12):4457-4469

3. Xing C, Ma S, Wu YC, Ng TS (2010) Transceiver design for dualhop non-regenerative MIMO-OFDM relay systems under channel uncertainties. IEEE Trans Signal Process 58(12):6325-6339

4. Wang $R, T a o M(2012)$ Joint source and relay precoding designs for MIMO two-way relaying based on MSE criterion. IEEE Trans Signal Process 60(3):1352-1365

5. Khandaker MRA, Rong Y (2012) Joint transceiver optimization for multiuser MIMO relay communication systems. IEEE Trans Signal Process 60(11):5977-5986

6. Day BP, Margetts AR, Bliss DW, Schniter P (2012) Full-duplex MIMO relaying: achievable rates under limited dynamic range. IEEE J Sel Areas Commun 30(8):1541-1553

7. Lin C, Tseng F, Wu W (2017) MMSE transceiver design for full-duplex MIMO relay systems. IEEE Trans Veh Technol 66(8):6849-6861

8. Rodriguez LJ, Tran NH, Le-Ngoc T (2014) Performance of fullduplex AF relaying in the presence of residual self-interference. IEEE J Sel Areas Commun 32(9):1752-1764

9. Bharadia D, McMilin E, Katti S (2013) Full duplex radios. In: Proceedings of the Hong Kong, China, Aug, ACM SIGCOMM, pp 375-386

10. Riihonen T, Werner S, Wichman R (2011) Mitigation of loopback self interference in full-duplex MIMO relays. IEEE Trans Signal Process 59(12):5983-5993

11. Shang CYA, Smith PJ, Woodward GK, Suraweera HA (2014) Linear transceivers for full duplex MIMO relays. Sydney, Australia, Feb, In: Proceedings of the Australian Communication Theory Workshop, pp 11-16

12. Shao Y, Dai Y, Gulliver TA, Dai X (2019) Transceiver design for multiple-input multiple-output full-duplex amplify-and-forward relay communication systems. IET Commun 13(1):66-73

13. Suraweera HA, Krikidis I, Zheng G, Yuen C, Smith PJ (2014) Low-complexity end-to-end performance optimization in MIMO full-duplex relay systems. IEEE Trans Wireless Commun 13(2):913-927 
14. Kang YY, Cho JH (2009) Capacity of MIMO wireless channel with full-duplex amplify-and-forward relay. In: Proceedings of the IEEE international symposium on personal, indoor and mobile radio communications, Tokyo, Japan, pp 117-121

15. Boyd S, Vandenberghe L (2004) Convex optimization. Cambridge University Press, Cambridge
16. Grant MC, Boyd SP (2015 Jun) CVX: Matlab software for disciplined convex programming, [Online]. http://cvxr.com/cvx/

Publisher's Note Springer Nature remains neutral with regard to jurisdictional claims in published maps and institutional affiliations. 